\title{
Enhancing and Testing Electrical Engineering Design Skills
}

\author{
W G Dunford \\ Department of Electrical and Computer Engineering \\ University of British Columbia \\ Vancouver V6T $1 Z 4$ \\ e-mailwgd@ece.ubc.ca
}

\begin{abstract}
Experience with various design related courses is described. In particular, the use of laboratory examinations to test design skills is discussed. Examples of design projects at both junior and senior levels are outlined.
\end{abstract}

\section{Introduction}

Traditional electrical engineering laboratory work has involved students working in pairs on a task which may or may not have been discussed in associated lectures. The task is often presented as a cookbook implementation with detailed procedures and test expectations. The learning outcomes from this process are that the students will be able to perform practical tests under time constrained conditions and report their findings in a logical and organized manner. There is some virtue in this, but unless care is taken over assessment there is ample opportunity for cheating and for some individuals to act always as recorders rather than taking an active part in the practical activity.

The experience of the author with various approaches to testing practical skills will be described and in particular the implementation of practical examinations will be discussed in detail.

\section{The Design Process}

There is a variety of opinions on what is really meant by good design. An electrical engineer tends to think of economic and reliability questions. Someone from marketing will think primarily about utility and aesthetics. A classic industrial problem is for the marketing department to decide on how a product should look without consulting the manufacturing department. This can lead to designs which are impossible or uneconomic to make.

From a utilitarian standpoint a product which performs well and is cheap to make and reliable is a good design. A good industrial designer expects more than this. There should be an element of love. The user should actively want to use a well designed product and would spontaneously pick a well designed product over one which is simply utilitarian. Often design enhancements can be made at little cost. For example, a pen with a circular cross section is quite usable. One with a more triangular cross section which nestles between the fingers is a joy to use. It is this type of thinking which must be instilled in engineering students.

An important question is how much of the design process we should expect an ECE student to be able to do on graduation. Buckminster Fuller referred to the "Leonardo type" as someone akin to Leonardo da Vinci. 
This renaissance person would be skilled at all aspects of a project from artistic conception through to engineering implementation. Most students do not fall into this category, so it must be decided how far to try to take them. At one extreme it could be argued that in future all electronics are likely to be implemented on a chip and that an electrical or computer engineer would be surrounded by other specialists and technicians to do things like designing packaging and building circuits. This type of engineer would be a pure theoretician and might actually be completely dispensable. At the other end of the scale it might be felt that we should produce engineers as close as possible to the Leonardo type. People who can graduate and then start a company in the basement, immediately building on all the practical and theoretical background they have gained in university. In the ECE department at UBC we have used this latter type as the model and some of the tools used to achieve this will be described here. There are some variations on the theme such as a totally project based approach, but this paper focusses on the program taken by the majority of students.

\section{Prior Learning Assessment}

In senior years there is reasonable knowledge of what students have done in earlier years. Second year students have been exposed to a small amount of laboratory work in first year but their skills are widely divergent. There are almost no students who have spent happy evenings building amplifiers or amateur radio transmitters, as was common 30 years ago. Some students may have taken high school electronics or come through another institution such as the British Columbia Institute of Technology (BCIT). However, recent experience has shown that $50 \%$ of the second year students have no real practical skills at all. Not only have they never touched a soldering iron, their facility with something as basic as a screwdriver is rudimentary. These students may be able to comment on design from the viewpoint of a user, such as whether a piece of software or a cell phone is easy to use. They are certainly not in a position to comment on how a design might be improved or that improvement implemented.

\section{Imparting Basic Skills}

In response to the decline in incoming practical skills and feedback from employers that UBC students were too theoretical, an effort was started about 15 years ago to make sure that every UBC ECE graduate could demonstrate some competence in practical design and using the tools of the trade. It had previously been possible for someone who was not practically oriented to make sure that they were always involved with the theoretical side of things. They would always be the partner that recorded results and did calculations rather than actually touching the oscilloscope.

All regular option $2^{\text {nd }}$ year ECE students must now achieve the following:

1. Demonstrate a knowledge of workshop and electrical safety.

2. Build a simple box to house an electronic project. This involves cutting, bending and drilling sheet metal.

3. Design and simulate an electronic circuit to meet given criteria.

4. Layout a printed circuit board (PCB) to implement the designed circuit.

5. Solder the components into the PCB.

6. Complete the assembly of the $\mathrm{PCB}$ into the box.

7. Demonstrate the project and write a 
report on the design.

8. Perform a certain number of standard electronics experiments, which are recorded in a laboratory notebook.

9. Demonstrate individual competence via a practical laboratory examination.

10. Contribute to a group project report on a current electrical or computer engineering topic.

\section{Organization of $2^{\text {nd }}$ Year Work}

At present the second year laboratory work is concentrated into a single course consisting of 1 lecture and 3 laboratory hours per week. Students are not assigned to groups but can work as they please. They are given keycodes and can access the laboratory 24 hours a day. All laboratory material is available via a WebCT site which is accessible via the Internet and computers at the benches. Components are supplied via an open stock, but students must buy their own breadboards. This system has been abused by a minority of students and is at present under review. Teaching assistants are present during the assigned times and are responsible for marking the notebooks. Students are given milestones for the completion of experiments in a particular module. The emphasis is on proper record keeping and analysis rather than writing up laboratories in a formal way.

The history of second year laboratory work is that it used to be included with lecture courses as set piece experiments. The marks for this portion were generally high and there were many cases of students passing courses largely on the basis of the laboratory marks. It was decided that moving all laboratory work into a separate course would avoid this problem and allow the coordination of all laboratory work under one person. A lecture was included so that there was an opportunity each week for all students to be brought together to be given information on upcoming experiments. This slot is not used every week, but makes a convenient time for other related material such as videos on industrial applications to be shown.

Another course which was introduced at about the same time was a group project. Here students are assigned to a group and a faculty member. The main deliverable from this course is a report to be presented in both oral and written form. This is usually a paper study on a topic in the research area of the supervisor. For example, "The Design Requirements of a Fly by Wire Aircraft Control". The intention is that students will have to delve into a topic and get some grasp of real engineering problems in time to help them choose options in later years. In the process they will also have to work with a group of people they may not know or like, much as in the real world.

Part of this course is one lecture a week, which is used for a variety of topics. Typically there will be a lecture or two on group work and lectures by visitors from industry on subjects such as the management of an engineering consulting company.

This course was started partly in response to suggestions that our graduates needed improved communication skills. There is now a similar course in the common first year which introduces topics in different engineering disciplines.

\section{Junior Projects}

Since the second year laboratory time is not strictly structured it was decided to add some design project work alongside some more conventional experiments. Some self initiated projects have been allowed, but due to the large numbers involved all students have been 
encouraged to work on the same assigned project. Since there are other opportunities for group work the junior project is an individual effort. This does lead to copying problems, but there are checks in place to reduce this.

This year the topic was the "Electronic Lock". In the first term a standard box was constructed and a PCB of standard dimensions allowed. In recent years we have standardised on "CircuitMaker" as our simulation package with the companion "Traxmaker" as the PCB layout software.

The specifications for the first term were that a simple digital code had to be generated and transmitted by an infrared link. This hardware was to be implemented in discrete logic and mounted on a PCB. A receiver was to be designed and constructed on a breadboard, which would light indicator lamps in response to the received signals.

This project thus had a number of planned learning outcomes such as:

1. Demonstrate ability to use hand tools and test instruments safely.

2. Solder components to a through hole board and describe the precautions necessary.

3. Simulate a simple mixed analog/digital circuit and design a related PCB.

4. Describe how ambient light can affect an optical communication system and how this can be accommodated in the electronic design.

In the second term a related project was undertaken. This required building a new PCB to accommodate a $68 \mathrm{HC} 11$ microprocessor and related interface circuitry to produce a more sophisticated transmitter module. The receiver was implemented with another $68 \mathrm{HC} 11$ development board already used in related experiments.

The specifications were now expanded to make the system more secure. For example the new system had longer code lengths and required the use of a random number generator to achieve some encryption. The output device was changed from lamps to a 7 segment display.

\section{Project Assessment}

With small numbers it is possible to assess all the projects in one session. With the 7 sections required here the assessment has to be spread over several periods. Various strategies have been tried, such as giving students set times to demonstrate, but enforcement is a problem. We now require students to arrange a demonstration with their assigned teaching assistant before a certain date and sometimes a mark incentive is given for early submissions.

A key element of the assessment process is that students are not given numerical marks at the time of assessment. They are given letter grades for each of a set of completed elements. These are as generic as possible, but sometimes there are achievements of particular relevance to a certain project.

An example of this check list is:

\section{Construction Type}

A - No circuit

B - Circuit on Veroboard or equivalent

C - Printed Circuit implementation

\section{Construction Quality}

A - Poor attention to detail (eg multiple components soldered together with long leads) B - Implementation could be improved (eg untidy wiring or PCB mistakes) $\mathrm{C}$ - Very neat implementation. 
Design

A - Little understanding of the problem or oversimplified.

B- A good attempt but many details missing. C- Very good attempt but some details missing.

D- All criteria met.

E- Design particularly well conceived such as very economical use of parts .

\section{Performance}

A - Non functional

B - Some circuit activity, but not close to requirements.

C - Operational, but some aspects could be improved.

D- Meets all specifications under all operating conditions.

E- Exceeds specifications - exceptional performance.

\section{Understanding}

A - No understanding of the circuit operation, such as inability to identify the individual integrated circuits.

B - Little understanding of what was being attempted.

C - Able to explain basic principles of design but unsure of some details.

D - Coherent explanation of design without all details

E - Coherent explanation of the design with details such as how frequency was decided in terms of device specifications.

F - Masterful explanation of design and its operation.

\section{Collaboration}

A - Much helped by others.

B - Worked with others but made a significant contribution.
C - Entirely individual effort.

\section{Overall Impression}

A- Nothing worthwhile

B- Unsatisfactory, some effort but many problems

C - Poor, only some requirements met.

D - Fair, meets some of the requirements but could be much improved.

E- Average effort, possibly some aspects incomplete.

F- Good but room for improvement.

G- Very good, but one aspect might be deficient.

$\mathrm{H}$ - Excellent, only very minor improvements possible.

J- Outstanding piece of work, one in a hundred.

This list was used by several different markers and has given fairly consistent results in terms of the averages of different groups. The letter grades are entered into a spreadsheet and converted to numerical grades by applying conversion factors. These factors can be adjusted according to the overall performance of the whole class.

Cheating is a potential problem, with parts of the project being shared by different people. At one stage the projects were collected as they were marked, but dealing with the large number of circuits was more trouble than it was worth. We now take as many precautions as possible, including requiring student names to be etched on to PCBs.

\section{Laboratory Examinations}

The second year laboratory examination is designed to test the practical knowledge and design skills of individual students. Students will typically take three such examinations during the year, partly for some to get over the 
shock of the first one and partly to compensate for possible differences between tests. With the number of students involved we need to run at least five 2 hour sessions, each with its own examination.

The format of these examinations has varied slightly over the years and now consists of two questions. The first is a written question and the second is a design and build question. The students may have any books and aids they wish, apart from devices which communicate with other people. Experience has shown that students who do badly in this test are almost beyond help. They have typically not participated well previously and forget such obvious things as connecting the power supply to the integrated circuits. These are just the sort of people the test is intended to catch and many of them only fail the first test. The overall failure rate in the course is typically less than $10 \%$.

A typical set of test questions would be:

1. Two channels of an oscilloscope are connected in parallel to a square wave source. The display from one channel is correct but from the other the pulses are not square. Give possible reasons for this.

2. Design and build a circuit which will take a $\pm 5 \mathrm{~V}, 1 \mathrm{kHz}$ square wave as an input and produce a $\pm 5 \mathrm{~V}$ triangular wave as an output.

The second question would typically be built from a set of parts and data sheets supplied and it would be left running at the end of the test.

\section{Test Assessment}

Generally several teaching assistants and other staff are involved in marking different sections after the students have left the room.
The first question is normally given 5 marks and most students will get full marks, although some will miss the point. The second question is the main point of the test and there is room for subjective assessment. We are using the following assessment descriptions to arrive at a mark:

For the display element

9 - perfect display.

8 - minor problem such as a poorly chosen scale

7 - basically correct, but perhaps poorly triggered or with some noise

6 - more major problem such as wrong frequency or voltage

5 - some resemblance of what is required, eg major distortion

4 and under for charitable use only. This really implies that almost nothing has been achieved.

\section{For the theory}

6 - clear explanation of what was intended. This would be the right design even if it was not implemented.

5 - the right general idea but some details missing

4 - some understanding of what was needed but few details

3 - almost nothing right

2 and under totally unsatisfactory. This would include people who had built a correct circuit but not written anything down about it. 


\section{Senior Projects}

In the fourth year there are two project courses. One is a combination of a previous project course and a report writing course. The marking of this course is a little complex, but the design element consists of students working alone, or in small groups with a faculty supervisor. Projects can be generated by the supervisor or the student. In this course everything is a matter of free choice. Some supervisors will have 5 students, others none. There is a course coordinator, but apart from general guidelines the conduct of the projects is left up to individual faculty members. In some cases students will work in industry with an industrial supervisor. Potential intellectual property difficulties are avoided by defining these students as taken part in unpaid coop work terms.

The quality control of the projects might be improved, but politically it is difficult for the course coordinator to spend too much time peering over the shoulders of colleagues.

\section{Senior Group Project}

The second fourth year project course is specifically a group project. In the early days of this course all groups were assigned a particular task in the form of a competition. This approach introduces a competitive element which students like and simplifies judging because all projects have the same goals, such as a robot getting to the centre of a maze quickly.

Some problems with this approach are that students are not required nor allowed to think of their own real world problem to be solved. There is also the assumption that the device which wins the competition should also get the best mark. It is difficult to convince students that the device which came in last might be considered the best because its battery life is twice that of any of the others.

More recently this course has evolved into a more formal design course with more emphasis on a structured design process. The students are assigned groups and a teaching assistant or instructor as their primary technical contact. This course runs three times a year and one managerial problem is that students add and drop at the last minute, depending on the job situation or their dislike of the students they have been assigned to work with. This is in contrast to the second year where there is no choice and students progress in lock step.

There are some distinct milestones:

1. Submit two project proposal outlines and a group structure. In particular the group leader should be chosen with reasons given for the choice.

2. After discussions with the advisor submit a detailed proposal for the chosen project with Gantt chart.

3. Submit a progress report and attend an interview to review this.

4. Submit a written report on the project with a complete costing.

5. Demonstrate the project and submit a video describing the project.

The budget for these projects is $\$ 400$ per group and assigned bench space and equipment is available 24 hours/day. The aim is to come as close as possible to an industrial design process with specifications being generated and adhered to. In the demonstration factors such as reliability and presentation receive particular attention. A device which has a battery hanging off the back via duct tape will certainly get a lower mark than which has a well designed battery compartment. 


\section{Faculty Interaction}

A fine balance must be struck between the advisor trying to impose a solution and letting the group flounder with no solution. It is the responsibility of the group leader to keep the project on track, but typically some outside help is required to achieve this. Faculty are always available to answer questions, but weekly meetings between the group and advisor are required for the first month to make sure that everything is on track. As far as possible a Problem Based Learning approach is used where students are advised of sources and encouraged to come to their own conclusions about the quality of their designs. Certainly, it is often difficult to avoid simply telling the group how you think it should be done. This is often what the group really wants to hear.

\section{Assessment}

There are nominally separate mark components for the different elements such as the mid term and final reports. However, it is clear that the performance in the final demonstration will be the last impression that the judging committee has and typically the other elements are only looked at critically if there is some disaster in the final demonstration.

The final group marks are determined at a meeting of all faculty and teaching assistants associated with the course. Generally each committee member decides an order in advance and notes are compared. There is usually good agreement on the order without the need for much discussion and the numerical marks must then be determined. This is usually done from a starting point of maximum and minimum, based on experience with previous projects. The intermediate marks are then determined.

There is clearly a subjective element here but the students realize that we do our best and that the marks are non negotiable.

\section{Self and Peer Evaluation}

Marks for individual students are determined entirely by the student group unless there is substantial disagreement. A self and peer evaluation scheme is used in the final interview. Students are simply asked to mark each member of the team, including themselves, with a score between 0 and 9, representing total contribution over the duration of the project. Some attempt was made to obtain a more detailed picture, but it did not seem worth the effort.

The vast majority of the groups award each other almost identical marks, meaning that the group mark is given to all the members. In cases where this is not true it is sometimes necessary to look more closely at individual activities such as scrutinizing the notebooks that all students are asked to keep. It is possible for one group member to fail on a project considered first class over all.

Self and peer evaluation is a difficult process for students. They do not like to confront each other. Although we recommend that they do this weekly we have not required it. We do make them do it twice, once in the middle of the term when it is not counted and once at the end of the term when it is.

\section{Typical Senior Group Projects}

Many groups are interested in some sort of robot and these include walking robots and robot surveillance systems, with video links via the internet. The main problem is that the mechanical skills are lacking and joint 
projects with mechanical engineering students would be very beneficial. This has often been discussed, but at present the two departments do not have compatible timetables.

Another group of popular projects are those directly focussed on using a computer. These range from games to packages for drawing and for managing video and audio files. There are other projects which might be described as aids to the disabled, including Braille translators and pill dispensers.

A common weakness in most projects is the way power is managed and the mechanical finish. The projects that are rated very highly tend to be those which have attended to those details. For example, something that will run efficiently from a single battery rather than running for 2 minutes from three different sources.

\section{Project Fair and Competitions}

In common with many universities the UBC Engineering Physics program has had a annual project fair for many years. This provides a focus for project work and an opportunity for industry to see what is going on at the university, potentially meeting future employees. The first ECE project fair was held in 2003 with the financial support of the Kaiser Educational Foundation. A junior and senior prize was awarded and the judging was in the hands of a visiting industrial panel. The focus of the judging was on the overall design of the device and it was valuable to both students and faculty to see what factors caught the eyes of people from the commercial world.

An interesting comparison can be made between mechanical and electrical students at $\mathrm{UBC}$ and there is anecdotal evidence that a similar situation exists at other universities.
Mechanical engineers seem much more oriented to design competitions. They are effectively required to participate in an external competition of some sort, such as the human powered submarine.

By contrast, electrical engineers treat competitions with apathy, even when the prizes seem generous. Often it is simply necessary to retain something already built in a course, but this seems to be too much trouble. International competitions run by the IEEE on topics such as fuel cell electrical interfaces have generated few if any entries and none from Canada. Most people would say that design competitions are a good idea, but students appear to believe that it is not a good use of their time. The truth may be that engineering students do not have enough spare time.

\section{Graduate Design Projects}

There is an academic bias against graduate projects which produce something which looks like an industrial product. The NSERC IPS program has been very successful in the past at getting students into industry but it has been difficult to convince committees that a new way of controlling a commercial power supply really counts as research. Some equations describing aspects which are not of practical interest are what is expected.

Another student has just started on a project which is part of the Auto21 NCE. This is specifically a design oriented project and it remains to be seen whether this will have a sufficient "contribution". The goal is to design an electrical machine which is optimized for regenerative braking in a conventional vehicle. It is hoped that this will actually be built, but we may have to be content with finite element simulations. 
In general the most difficult projects to justify in research terms seem to be system optimization problems. A real engineering problem has been solved, but there is not a detailed analysis of an individual part. In the process the student is certainly exposed to commercial design requirements and will probably be quickly employed by a company if not by a university.

\section{Conclusions}

Design skills are enhanced and tested at all levels by a combination of laboratory examinations and project assessments. Where possible an industrial focus is introduced. Students are assessed on both their individual skills and their ability to produce a successful result through working as part of a team.

Detailed costs have not been presented and at present it is not easy to identify costs associated with individual course. There has also not been a detailed analysis of the learning outcomes of our present program compared to the time when things were done differently. Anecdotally there seem to be fewer negative comments from industry but there are other factors which have not been considered such as a switch from a 5 to 4 year program and the lower intake marks as we expand our student numbers. 